\title{
Selective Outcome Reporting (SOR) in Pediatric Dentistry restorative treatment randomized clinical trials - A meta-research.
}

Rokaia Ahmed Elagami

Universidade de São Paulo: Universidade de Sao Paulo

Tamara Kerber Tedesco

Universidade Cruzeiro do Sul

Claudio Mendes Pannuti

Universidade de São Paulo: Universidade de Sao Paulo

Gabriela Seabra da Silva

Universidade de São Paulo: Universidade de Sao Paulo

Mariana Minatel Braga

Universidade de São Paulo: Universidade de Sao Paulo

\section{Fausto Medeiros Mendes}

Universidade de São Paulo: Universidade de Sao Paulo

Daniela P Raggio ( $\nabla$ danielar@usp.br)

Universidade de São Paulo https://orcid.org/0000-0002-0048-2068

\section{Research Article}

Keywords: Randomized controlled trials, Selective reporting, Restorative treatment, Pediatric dentistry

Posted Date: December 9th, 2021

DOI: https://doi.org/10.21203/rs.3.rs-1048206/v1

License: (9) This work is licensed under a Creative Commons Attribution 4.0 International License.

Read Full License 


\section{Abstract}

\section{Background}

Selective outcome reporting (SOR) is a type of bias that occurs when the primary outcome of a randomized clinical trial (RCT) is omitted or changed prospectively. We evaluated the prevalence of SOR in RCTs on restorative caries treatment in primary teeth.

\section{Methods}

We conducted an electronic search on ClinicalTrials.gov and the World Health Organization (WHO) platform up to April/2021. We included RCT protocols that tested restorative treatments in primary teeth and excluded any protocol that has not resulted in at least one publication in a peer-reviewed scientific journal. The Chi-square test was used to detect the association between SOR and other variables $(a=5 \%)$.

\section{Results}

Out of 294 potential protocols, thirty were included. We found 34 corresponding publications and had the one that seemed to report the primary outcome and longest follow-up, resulting in 30 publications. SOR was observed in $53.3 \%(n=16)$ of the published trials and was significantly associated with the discrepancy in the follow-up period $(p=0.017)$.

\section{Conclusion}

There is a considerable prevalence of selective outcome reporting (SOR) on restorative trials in primary teeth. Properly pre-registered protocols and assessing them for the peer review processes will help decrease SOR.

\section{Practical implications}

Restorative treatment trials in primary teeth that selectively modify outcomes of interest have been shown to distort the treatment effect. Practitioners should avoid using restorative treatments based on misleading results in clinical practice.

\section{Introduction}

Dental caries in primary dentition is considered the 10th most common oral condition, affecting 621 million children worldwide (Kassebaum et al., 2015). Moreover, untreated carious lesions in children have a negative effect on oral health-related quality of life (OHRQoL) (Freire et al., 2018), and can provoke growth and developmental problems (Sheiham A 2006), toothache, and difficulty in sleeping (Perazzo et al., 2017). Well-designed and conducted Randomised Clinical Trials (RCTs) are considered the best level of evidence for interventional studies (Concato et al., 2000). The validity of clinical trials could be 
affected if they present biases (Suñé et al., 2013), and selective outcome reporting (SOR) is considered one of the potential biases that can overestimate the study's effect (Braakhekke et al., 2017).

"Spin" or misrepresentation in scientific literature occurs when the authors distort the interpretation of results and mislead the readers. In addition, the term "spin" is used when researchers try highlighting a specific benefit of experimental treatment despite a statistically non-significant difference for the primary outcome (Boutron et al., 2010). Moreover, studies had shown that the statistically significant results had higher chances to be published than non-significant results (negative), leading to publication bias. Furthermore, some researchers tend to alter their results to focus on particular conclusions (Contopoulosloannidis et al., 2006). When only positive or "spin" results are available, clinical decision-making can be jeopardized using Evidence-Based Practice.

Since 2005, the International Committee of Medical Journal Editors (ICMJE) has declared that all clinical trials must be registered before the first participant's enrollment. Consequently, prospectively registration of RCT's protocols will help to diminish the reporting bias (De Angelis et al., 2004; Smaïl-Faugeron et al., 2015). Transparency between the planned and published outcomes will prevent waste of time, effort, and money (Chalmers \& Glasziou 2009 ). Two essential guidelines have been introduced to enhance transparency. First, the SPIRIT recommendation (Standard Protocol Items: Recommendations for Interventional Trials) in 2013, which has a 33 items checklist, was concerned about content rather than the design of RCT protocols (Chan et al., 2013). Second, the CONSORT guidelines (Consolidated Standards of Reporting Trials), was also introduced to improve the reporting quality for RCTs (Moher et al., 2012; Boutron et al., 2008).

Prospective trial registries can help to reduce the SOR. Furthermore, SOR could be prevented through joint attempts from the editors, authors, and reviewers. Editors and reviewers should request the protocol registry to assess the discrepancies in the data, time frame, or outcomes, and the journals' adherence to the reporting guidelines. Moreover, the authors should comply with the reporting guidelines and report any deviations from the protocol on their publication (Munafò et al., 2017; Nosek et al., 2018).

A study found that out of 78 examined in general oral health journals, $32(41 \%)$ required or recommended trial registry (Smaïl-Faugeron et al., 2015). Another study has identified 27 studies (55\%) with high rates of SOR in clinical trials of dental implants among the 49 available protocols (Sendyk et al., 2019). As mentioned before, SOR might distort the evidence available for the decision-making of the clinician. Moreover, the consequences of this type of bias could be a significant threat. To our knowledge, there has been no previous evaluation of selective outcome reporting in RCTs in the field of Pediatric Dentistry.

Thus, our primary aim was to examine the selective outcome reporting by comparing information from records with the publications of RCTs concerning restorative dentistry in primary teeth. The secondary goals were a) evaluate the introduction of new secondary outcomes (i.e., an outcome that was not described in the registry and that was introduced as secondary in the publication); b) discrepancies between the trial registry and publication regarding study start date, number of arms, follow-up period, 
and sample size; c) other types of discrepancies; d) association between SOR and the timing of registration, type of sponsorship, type of study design and discrepancies of follow up.

\section{Material And Methods}

This study is a meta-research and was registered in the Open Science Framework platform (10.17605/OSF.IO/8H5PA). The research question for the present study is: "From previous registered RCTs in restorative care for primary teeth, how many have a selective outcome reporting in the published paper?".

\subsection{Search strategy}

We searched for RCTs protocol registries that dealt with restorative treatment in primary teeth. We conducted the search in the ClinicalTrials.gov and WHO platform, up to April/2021, with no registry time or language restrictions. The search strategy was: ("dental caries" OR decay OR caries OR carious) AND ("primary teeth" OR "primary tooth" OR deciduous OR "primary dentition" OR children OR child OR infant). We limited the results on clinicaltrials.gov only for "unknown and completed status" protocols. We used only two keywords for the WHO International Clinical Trials Registry platform: "dental caries" and "primary teeth." We checked the results of both searches to find and eliminate duplicates.

\subsection{Study selection}

Two researchers independently screened the title and abstracts of the registered protocols for eligibility. The inclusion criteria were: (a) RCT with two or more arms; (b) study evaluating any restorative treatment on primary teeth. We did not include observational studies (cohort, case-control, and cross-sectional) and case series. We excluded any protocol that has not resulted in at least one publication in a peer-reviewed indexed scientific journal. We searched the corresponding published article for each included protocol by finding the reference(s) of the publication on the registry. We searched on PubMed and EMBASE using the principal investigator's name and protocol's keywords when it was not available. If we could not find any publication, we searched Google Scholar with the record number. If we found more than one publication, we extracted the data from the one that has the alleged primary outcome. If we found more than one primary outcome publication, we extracted the data from the registry with the longest follow-up period. When no publication was found, we contacted the principal investigator, and we have excluded the study protocol in case of no response. A third researcher was responsible for resolving any disagreement.

\subsection{Data extraction}

Two researchers independently extracted the data for each included protocol and their corresponding publication. Any disagreements were solved by the third researcher. We registered all the extracted data from the included protocols and publications in a standardized form. 
First, we extracted some information from the protocols, such as: registry number, name and country of the principal investigator, primary registry date (and last time updated), study start date, and the completion date. To define if the study was prospectively or retrospectively registered, the registry timing was analyzed. If the protocol was registered before the enrollment of the first participant, it was considered a prospective registration. If authors recorded the protocol after the enrollment of the first participant, it was considered a retrospective registry. In addition, we identified if there were any core changes in the protocol, using the history of changes in ClinicalTrials.gov. We also extracted data about the sponsorship (industrial, commercial, or personal funding), number of arms and intervention, RCT design, sample size, and follow-up time. We also collected the number, nature, and timeframe for each primary and secondary outcomes.

We gathered the number of publications, the journal's name, the journal's impact factor (Journal Citation Reports - 2020), study start date as mentioned in the publication, and paper publication date. We also recorded the sponsorship of the article (institutional, commercial, or incompletely identified). We classified the impact factor of the journals as "high impact "or "low impact," using the median of the area as the cut-off value. We recorded if the author had notified the registry number. We also checked if the author declared any changes in the protocol and collected data for the number of arms and interventions, sample size, a priori sample size estimation based on the primary outcome. For the outcome, we recorded the number, nature, time frame, and whether the primary or secondary outcomes and if they have shown statistical significance.

After collecting all data from the protocols and publications, we compared the data to extract the discrepancies. We identified the SOR according to a modification of Chan et al., 2004: a) primary outcome in the registry reported as secondary in the publication (primary outcome downgrade), b) secondary outcome in the registry reported as primary in the publication (secondary outcome upgrade), c) a new primary outcome (i.e., an outcome that was not described in the registry) introduced in the publication, d) primary outcome in the registry omitted in the publication, e) discrepancy in the primary outcome time frame (i.e., the timing of assessment of primary outcome differs between registry and publication). When the registry has more than one primary outcome, we consider the primary outcome in the publication according to which the sample size calculation was based, and all the other outcomes considered secondary outcomes. In addition, we investigated other discrepancies between the protocol and published articles, such as discrepancies in the study start date, study design, arms, sample size, and sponsorship (commercial versus non-commercial).

\section{Data Analysis}

We used the Jamovi Version 1.2 software to perform the statistical analysis. Quantitative variables were expressed as the mean and standard deviation, and qualitative variables were expressed as the frequencies and percentages. We used the chi-square test to detect the association between the SOR and the following variables: discrepancy in the follow-up period (yes or no), the timing of registration 
(retrospective or prospective), the type of sponsorship (no sponsorship, commercial or non-commercial), and study design. The significance level for all analyses was set at $5 \%$.

\section{Results}

Our search strategy identified a total of 294 protocols from both clinicaltrial.gov and the WHO platform. After screening the titles and abstracts, we included 74 protocols. Forty-four protocols were excluded as we could not find their corresponding publications. Eventually, 30 studies were eligible and included in the review (Figure 1).

Table 1 shows the characteristics of the included protocols. The majority of the protocols were funded by the Pl's institution and registered retrospectively. About core changes in the protocol, the majority had no listed modifications. Furthermore, most of the trials had two arms with parallel-group design. The primary outcome time frame ranged from 12-48 months, and more than half of the trials were reported with one primary outcome.

Regarding the characteristics of the corresponding publications (Table 2), the trials were published in 19 different journals, mainly in pediatric dentistry and medicine fields. The majority of the protocols have been identified with one publication.

Twenty-seven (90\%) studies cited the registry number in their publications. Twelve (40\%) showed statistically significant findings for a minimum of one primary outcome. Moreover, only two publications declared that there was a deviation from the registered protocol. Regarding sample size, the majority of the authors calculated it based on the primary outcome. The primary outcome time frame ranged from 648 months.

We observed selective outcome reporting in 16 (53.3\%) of the trials (Table 3). Among discrepancies between the protocols and publications, the most common reason for SOR was a discrepancy in the primary outcome time frame $(n=12,40 \%)$. We identified other discrepancies as to the following: primary outcome downgrade $(n=6,20.0 \%)$, secondary outcome upgrade $(n=4,13.3 \%)$, primary outcome reported in the protocol that omitted in the publication $(n=1,3.3 \%)$, and new primary outcome introduced in the publication $(n=3,10 \%)$. Seventeen $(56.7 \%)$ trials were reported with new secondary outcomes in addition to various outcome discrepancies.

Table 3 displays further discrepancies between protocols and publications. Thirteen studies (43.3\%) reported a discrepancy in the study start date. Only $3(10 \%)$ trials reported a discrepancy in the number of arms. Eleven trials had a discrepancy in the follow-up period, with seven decreasing and four increasing. Thirteen trials reported the non-commercial difference in sponsorship, and two presented a commercial discrepancy between the registry and publication.

Table 4 shows the association between SOR and discrepancies in the clinical trials. SOR was only associated $(p=0.017)$ with the discrepancy in the follow-up period. We calculated the median of journals' 
impact factors (2.757) according to (Journal Citation Reports - 2020) and related the SOR to high-impact or low-impact factor journals. The impact factor of the journals ranges from (1.065 - 4.379). We found that SOR existed in 4 (25\%) of the three journals with high impact factors (Clinical Oral Investigations, Journal of Dentistry and Caries Research). Also, SOR existed in 12 (75\%) of the ten journals with low impact factor (Archives of Oral Biology, Quintessence International, Brazilian Oral Research, Pediatric Dentistry Journal, Laser in Dental Science, Journal of the Dental Association of Thailand, JDR Clinical \& Translational Research, Journal of Clinical Pediatric Dentistry, Journal of Dentistry for Children and Alexandria Dental Journal). There was no statistically significant difference between the SOR and impact factor of the journals $(p=0.156)$.

\section{Discussion}

This study is the first meta-research that focuses on SOR in Pediatric Dentistry restorative treatments and has shown a high prevalence of SOR. One systematic review in pediatric dentistry (Tedesco et al., 2018) showed that few good quality studies exist regarding the management of dentin caries. Most have a high risk of bias to provide enough evidence to recommend the best treatment option strongly. Selective outcome reporting (SOR) might distort the evidence available for the decision-making of the clinician. Moreover, the consequences of this type of bias could be a major threat. To our knowledge, there has been no previous evaluation of selective outcome reporting in RCTs focusing on restorative treatment in the field of Pediatric Dentistry. The suggested policy was trial registration with a pre-specified list of all the outcomes and transparent reporting of any changes afterward. Another attempt to improve the outcome reporting is followed by some journals (Kirkham et al., 2010) that have announced that each author has to declare that the manuscript is an honest, accurate, and transparent account of the study, and no critical aspect has been omitted.

The prevalence of selective outcome reporting is still high and has been reported by the dental and medical fields (Sendyk et al., 2019; Chan et al., 2004; Fleming et al., 2015; Aggarwal et al., 2019; Mathieu et al., 2009). Similarly, our results proved the discrepancies between the registered protocols and published articles in restorative treatment for primary teeth. We observed SOR in 53.3\% of the included trials, further information regarding the reasons of SOR in each included trial found in Online Resource1. Our results had become similar to other studies in dental and medical literature, such as $49 \%$ in surgical intervention trials (Hannink et al., 2013), 48\% in trials of osteoarthritis (Krsticevic et al., 2017), more than $55 \%$ in trials of dental implants (Sendyk et al., 2019), and 53.8\% of the prospectively registered psychotherapy trials (Bradley et al., 2017). A range of $40-62 \%$ has been reported in studies that evaluate publication bias and reporting bias in RCTs (Dwan et al., 2008), where at least one outcome is omitted, introduced, or changed. Empirical research pointed out that the positive or statistically significant results have more chances to be published, and the negative results could be rejected (Dickersin et al., 1987). Thus, we suggest that the editors could accept the publication of negative results considering that the authors followed the correct methodology. 
In our study, the primary outcome time-frame discrepancy was the most common among the trials reported with SOR. A recent study (Hannink et al., 2013) emphasized that $33 \%$ of their trials presented a primary outcome time-point discrepancy. In hematological malignancies, a previous study (Wayant et al., 2017) assessed RCTs published in hematology journals and showed a change in the timing assessment of the primary outcome by eight times (6.8\%). In contrast to the study of (Koufatzidou et al. 2019), which found an association between outcome reporting discrepancy and type of study design, we could not find any association. A possible explanation for this finding is that most of the assessed trials did not report the type of study design in their final publications.

Furthermore, we found a significant association between SOR and discrepancy in the follow-up period. Accordingly, the trials that differ in follow-up periods from their corresponding publications had presented with outcome bias. That was in agreement with one study (Rosati et al., 2016), as they found 13 RCT completed early without justification. In our study, we observed eleven studies that had a discrepancy in the follow-up period, two of them had no SOR. Seven studies changed the primary outcome time frame from the nine left, and two misreported their primary outcomes. That probably occurred when the authors decided to end the study before or after the completion of follow-up periods either if they had nonsignificant results ( $n=6$ studies) or if the favored result was found significant ( $n=3$ studies), affecting the study's reliability.

Although there was no association between SOR and the type of sponsorship, 33.3\% of the trials have non-commercial discrepancies. Consequently, some of these trials have shown one or more reasons for SOR. Likewise, other studies reported similar results (Braakhe et al., 2017; Fleming et al., 2015; Lancee et al., 2017). Also, $46.7 \%$ of the studies presented a discrepancy in sample size in our study, as observed in other studies (Koufatzidou et al., 2019; Rosati et al., 2016). Among these 14 trials, seven showed a decrease in sample size, seven trials have increased their sample size in the publication. In addition, a recent systematic review (Li et al., 2018) presented a range of $27 \%-60 \%$ of study discrepancies in sample size.

There was no association between SOR and registry timing (retrospective or prospective). However, the percentage of retrospectively registered trials was high (83.3\%), also observed in other publications (Fleming et al., 2015; Bradley et al., 2017; Farquhar et al., 2017; Su et al., 2015; Sendyk et al., 2021). Further, we verified that 14 trials with SOR were retrospectively published in 11 different journals (3 of them ICMJE member journals). Notably, since 2005 all the authors have to follow the ICMJE policy, which advocates registering the protocol with pre-specified and clear outcomes before starting the trials (enrollment of the first participant). Recent research (Mathieu et al., 2009) reported that the ICMJE requirements for protocol registration are not entirely followed. More data on Journal's Policies are depicted in Online Resource2.

We have expanded our search for two different platforms for increasing the potentially included protocols. Consequently, we had a limitation that most WHO platform trials have no "history of changes" function. Moreover, we noticed that inappropriate registration of the trials and unclear data about 
outcome measures could affect the recognition of outcome discrepancies, lowering the prevalence of SOR in our study. Another limitation to our study was that the protocol was excluded when we obtained no response from the principal author. This may have under- or overestimated the prevalence of SOR. Furthermore, most of the authors did not include the references of publications in the protocol after finishing the study. We overcame this problem by searching the registry number on Google scholar to find the corresponding publications. Hence, the importance of declaring the registry number in the publications contributes to recognizing them quickly for the readers and peer reviewers.

Despite all the attempts to decrease the selective outcome reporting and increase transparency, our findings indicated that the prevalence of SOR is still high in the trials focusing on restorative treatment in primary teeth, which are published in either high or low-impact journals. Evidence from the literature (Sendyk et al., 2019; Bradley et al., 2017; Rongen \&Hannink 2016; Shinohara et al., 2015) showed that even the prospectively registered trials had some sort of SOR mainly due to inadequate registered protocols. Thus, there is still a need to enhance trial registration awareness and properly pre-specify the outcomes. Also, any deviation from the protocol during the study should be reported in the publication. Further, during the peer-review process, it is mandatory that journal editors and reviewers have full access to the protocol of the study and compare it with the manuscript. Moreover, sponsorship funding should be declared correctly, and editors ensure that funded trials maintain their pre-specified registered protocol.

From 74 potential registries included in the methodological review, we could only find only 34 complete publications (two of them have 1-year results publication and two years, another two have published the primary outcome separate from the secondary outcomes). After contacting the authors, nine answered back with the reasons for not publishing the data. Among the answers from the authors, six manuscripts have been submitted to publication, one trial has no results, one trial was withdrawn, and one has been under analysis. It is possible that these trials had issues with the design, conduction, or even the results were negative. All information from an RCT must be available to reduce the research waste (loannidis et al., 2014). Well-designed and conducted RCT with negative results have to be published in high-quality journals, reducing publication bias. We hypothesize that one of the potential reasons that led authors to perform the SOR is related to publication bias (and it might not be deliberate), but related to the perceived or actual lack of interest from the research community (DeVito \& Goldacre 2019).

In this sense, we can conclude that selective outcome reporting in the pediatric dentistry restorative trials is high and might impact the clinician's decision-making process regarding restorative treatment in primary teeth.

\section{Conclusion}

The high prevalence of SOR in restorative treatment RCTs proved that this is a persistent issue. Therefore, properly pre-registered protocols and assessing them for the peer review processes will help achieve transparency between the registries and publications. 


\section{Declarations}

\section{Author contributions:}

Daniela Prócida Raggio, Claudio Mendes Pannuti, and Fausto Medeiros Mendes conceived the idea; Rokaia Ahmed Elagami, Tamara Kerber Tedesco, and Daniela Prócida Raggio collected the data; Rokaia Ahmed Elagami, Tamara Kerber Tedesco and Daniela Prócida Raggio analyzed the data; Rokaia Ahmed Elagami and Gabriela Seabra da Silva drafted the manuscript; Daniela Prócida Raggio, Mariana Minatel Braga, Fausto Medeiros Mendes, and Claudio Mendes Pannuti revised and gave final approval of the manuscript.

Competing Interests and Funding: None of the authors have any financial interest related to the article.

\section{Acknowledgments:}

This study was supported by the Coordenação de Aperfeiçoamento de Pessoal de Nível Superior (Coordination for the Improvement of Higher Education Personnel) (CAPES). Conselho Nacional de Desenvolvimento Científico e Tecnológico (National Council for Scientific and Technological Development) (CNPq) provides scholarship awards for research productivity in Brazil to Dr. Pannuti, Dr. Braga, Dr. Mendes, and Dr. Raggio.

The funders had no role in the design and conduct of the study; collection, management, analysis, and interpretation of the data; and preparation, review, or approval of the manuscript; or decision to submit the manuscript for publication.

\section{References}

Aggarwal, R., \& Oremus, M. (2019). Selective outcome reporting is present in randomized controlled trials in lung cancer immunotherapies. Journal of clinical epidemiology, 106, 145-146. https://doi.org/10.1016/j.jclinepi.2018.10.010.

Boutron, I., Dutton, S., Ravaud, P., \& Altman, D. G. (2010). Reporting and interpretation of randomized controlled trials with statistically nonsignificant results for primary outcomes. JAMA, 303(20), 20582064. https://doi.org/10.1001/jama.2010.651.

Boutron, I., Moher, D., Altman, D. G., Schulz, K. F., Ravaud, P., \& CONSORT Group (2008). Extending the CONSORT statement to randomized trials of nonpharmacologic treatment: explanation and elaboration. Annals of internal medicine, 148(4), 295-309. https://doi.org/10.7326/0003-4819-148- 20080219000008.

Braakhekke, M., Scholten, I., Mol, F., Limpens, J., Mol, B. W., \& van der Veen, F. (2017). Selective outcome reporting and sponsorship in randomized controlled trials in IVF and ICSI. Human reproduction (Oxford, England), 32(10), 2117-2122. https://doi.org/10.1093/humrep/dex273. 
Bradley, H. A., Rucklidge, J. J., \& Mulder, R. T. (2017). A systematic review of trial registration and selective outcome reporting in psychotherapy randomized controlled trials. Acta psychiatrica Scandinavica, 135(1), 65-77. https://doi.org/10.1111/acps.12647.

Chalmers, I., \& Glasziou, P. (2009). Avoidable waste in the production and reporting of research evidence. Lancet (London, England), 374(9683), 86-89. https://doi.org/10.1016/S0140-6736(09)60329-9.

Chan, A. W., Hróbjartsson, A., Haahr, M. T., Gøtzsche, P. C., \& Altman, D. G. (2004). Empirical evidence for selective reporting of outcomes in randomized trials: comparison of protocols to published articles. JAMA, 291(20), 2457-2465. https://doi.org/10.1001/jama.291.20.2457.

Chan, A. W., Tetzlaff, J. M., Altman, D. G., Laupacis, A., Gøtzsche, P. C., Krleža-Jerić, K., Hróbjartsson, A., Mann, H., Dickersin, K., Berlin, J. A., Doré, C. J., Parulekar, W. R., Summerskill, W. S., Groves, T., Schulz, K. F., Sox, H. C., Rockhold, F. W., Rennie, D., \& Moher, D. (2013). SPIRIT 2013 statement: defining standard protocol items for clinical trials. Annals of internal medicine, 158(3), 200-207.

https://doi.org/10.7326/0003-4819-158-3-201302050-00583.

Concato, J., Shah, N., \& Horwitz, R. I. (2000). Randomized, controlled trials, observational studies, and the hierarchy of research designs. The New England journal of medicine, 342(25), 1887-1892. https://doi.org/10.1056/NEJM200006223422507.

Contopoulos-loannidis, D. G., Alexiou, G. A., Gouvias, T. C., \& loannidis, J. P. (2006). An empirical evaluation of multifarious outcomes in pharmacogenetics: beta-2 adrenoceptor gene polymorphisms in asthma treatment. Pharmacogenetics and genomics, 16(10), 705-711.

https://doi.org/10.1097/01.fpc.0000236332.11304.8f.

De Angelis, C., Drazen, J. M., Frizelle, F. A., Haug, C., Hoey, J., Horton, R., Kotzin, S., Laine, C., Marusic, A., Overbeke, A. J., Schroeder, T. V., Sox, H. C., Van Der Weyden, M. B., \& International Committee of Medical Journal Editors (2004). Clinical trial registration: a statement from the International Committee of Medical Journal Editors. Lancet (London, England), 364(9438), 911-912. https://doi.org/10.1016/S01406736(04)17034-7.

DeVito, N. J., \& Goldacre, B. (2019). Catalogue of bias: publication bias. BMJ evidence-based medicine, 24(2), 53 https://doi.org/10.1136/bmjebm-2018-111107.

Dickersin, K., Chan, S., Chalmers, T. C., Sacks, H. S., \& Smith, H., Jr (1987). Publication bias and clinical trials. Controlled clinical trials, 8(4), 343-353. https://doi.org/10.1016/0197-2456(87)90155-3.

Dwan, K., Altman, D. G., Arnaiz, J. A., Bloom, J., Chan, A. W., Cronin, E., Decullier, E., Easterbrook, P. J., Von Elm, E., Gamble, C., Ghersi, D., loannidis, J. P., Simes, J., \& Williamson, P. R. (2008). Systematic review of the empirical evidence of study publication bias and outcome reporting bias. PloS one, 3(8), e3081. https://doi.org/10.1371/journal.pone.0003081. 
Farquhar, C. M., Showell, M. G., Showell, E., Beetham, P., Baak, N., Mourad, S., \& Jordan, V. (2017). Clinical trial registration was not an indicator for low risk of bias. Journal of clinical epidemiology, 84, 47-53. https://doi.org/10.1016/j.jclinepi.2016.11.011.

Fleming, P. S., Koletsi, D., Dwan, K., \& Pandis, N. (2015). Outcome discrepancies and selective reporting: impacting the leading journals?. PloS one, 10(5), e0127495.

https://doi.org/10.1371/journal.pone.0127495.

Freire, M., Corrêa-Faria, P., \& Costa, L. R. (2018). Effect of dental pain and caries on the quality of life of Brazilian preschool children. Revista de saude publica, 52, 30. https://doi.org/10.11606/S15188787.2018052000093.

Hannink, G., Gooszen, H. G., \& Rovers, M. M. (2013). Comparison of registered and published primary outcomes in randomized clinical trials of surgical interventions. Annals of surgery, 257(5), 818-823. https://doi.org/10.1097/SLA.0b013e3182864fa3.

Ioannidis, J. P., Greenland, S., Hlatky, M. A., Khoury, M. J., Macleod, M. R., Moher, D., Schulz, K. F., \& Tibshirani, R. (2014). Increasing value and reducing waste in research design, conduct, and analysis. Lancet (London, England), 383(9912), 166-175. https://doi.org/10.1016/S0140-6736(13)62227-8.

Kassebaum, N. J., Bernabé, E., Dahiya, M., Bhandari, B., Murray, C. J., \& Marcenes, W. (2015). Global burden of untreated caries: a systematic review and metaregression. Journal of dental research, 94(5), 650-658. https://doi.org/10.1177/0022034515573272.

Kirkham, J. J., Dwan, K. M., Altman, D. G., Gamble, C., Dodd, S., Smyth, R., \& Williamson, P. R. (2010). The impact of outcome reporting bias in randomised controlled trials on a cohort of systematic reviews. BMJ (Clinical research ed.), 340, c365. https://doi.org/10.1136/bmj.c365.

Koufatzidou, M., Koletsi, D., Fleming, P. S., Polychronopoulou, A., \& Pandis, N. (2019). Outcome reporting discrepancies between trial entries and published final reports of orthodontic randomized controlled trials. European journal of orthodontics, 41(3), 225-230. https://doi.org/10.1093/ejo/cjy046.

Krsticevic, M., Saric, D., Saric, F., Slapnicar, E., Boric, K., Dosenovic, S., Jelicic Kadic, A., Kegalj, M. J., \& Puljak, L. (2019). Selective reporting bias due to discrepancies between registered and published outcomes in osteoarthritis trials. Journal of comparative effectiveness research, 8(15), 1265-1273. https://doi.org/10.2217/cer-2019-0068.

Lancee, M., Lemmens, C., Kahn, R. S., Vinkers, C. H., \& Luykx, J. J. (2017). Outcome reporting bias in randomized-controlled trials investigating antipsychotic drugs. Translational psychiatry, 7(9), e1232. https://doi.org/10.1038/tp.2017.203.

Li, G., Abbade, L., Nwosu, I., Jin, Y., Leenus, A., Maaz, M., Wang, M., Bhatt, M., Zielinski, L., Sanger, N., Bantoto, B., Luo, C., Shams, I., Shahid, H., Chang, Y., Sun, G., Mbuagbaw, L., Samaan, Z., Levine, M., ... 
Thabane, L. (2018). A systematic review of comparisons between protocols or registrations and full reports in primary biomedical research. BMC medical research methodology, 18(1), 9. https://doi.org/10.1186/s12874-017-0465-7.

Mathieu, S., Boutron, I., Moher, D., Altman, D. G., \& Ravaud, P. (2009). Comparison of registered and published primary outcomes in randomized controlled trials. JAMA, 302(9), 977-984. https://doi.org/10.1001/jama.2009.1242.

Moher, D., Hopewell, S., Schulz, K. F., Montori, V., Gøtzsche, P. C., Devereaux, P. J., Elbourne, D., Egger, M., Altman, D. G., \& CONSORT (2012). CONSORT 2010 explanation and elaboration: updated guidelines for reporting parallel group randomised trials. International journal of surgery (London, England), 10(1), 2855. https://doi.org/10.1016/j.ijsu.2011.10.001.

Munafò, M. R., Nosek, B. A., Bishop, D., Button, K. S., Chambers, C. D., du Sert, N. P., Simonsohn, U., Wagenmakers, E. J., Ware, J. J., \& loannidis, J. (2017). A manifesto for reproducible science. Nature human behaviour, 1, 0021. https://doi.org/10.1038/s41562-016-0021.

Nosek, B. A., Ebersole, C. R., DeHaven, A. C., \& Mellor, D. T. (2018). The preregistration revolution. Proceedings of the National Academy of Sciences of the United States of America, 115(11), 2600-2606. https://doi.org/10.1073/pnas.1708274114.

Perazzo, M. F., Gomes, M. C., Neves, É. T., Martins, C. C., Paiva, S. M., Costa, E., \& Granville-Garcia, A. F. (2017). Oral problems and quality of life of preschool children: self-reports of children and perception of parents/caregivers. European journal of oral sciences, 125(4), 272-279.

https://doi.org/10.1111/eos.12359.

Rongen, J. J., \& Hannink, G. (2016). Comparison of Registered and Published Primary Outcomes in Randomized Controlled Trials of Orthopaedic Surgical Interventions. The Journal of bone and joint surgery. American volume, 98(5), 403-409. https://doi.org/10.2106/JBJS.15.00400.

Rosati, P., Porzsolt, F., Ricciotti, G., Testa, G., Inglese, R., Giustini, F., Fiscarelli, E., Zazza, M., Carlino, C., Balassone, V., Fiorito, R., \& D'Amico, R. (2016). Major discrepancies between what clinical trial registries record and paediatric randomised controlled trials publish. Trials, 17(1), 430.

https://doi.org/10.1186/s13063-016-1551-6.

Sendyk, D. I., Rovai, E. S., Souza, N. V., Deboni, M., \& Pannuti, C. M. (2019). Selective outcome reporting in randomized clinical trials of dental implants. Journal of clinical periodontology, 46(7), 758-765. https://doi.org/10.1111/jcpe.13128.

Sendyk, D. I., Souza, N. V., César Neto, J. B., Tatakis, D. N., \& Pannuti, C. M. (2021). Selective outcome reporting in root coverage randomized clinical trials. Journal of clinical periodontology, 48(6), 867-877. https://doi.org/10.1111/jcpe.13451. 
Sheiham A. (2006). Dental caries affects body weight, growth and quality of life in pre-school children. British dental journal, 201(10), 625-626. https://doi.org/10.1038/sj.bdj.4814259.

Shinohara, K., Tajika, A., Imai, H., Takeshima, N., Hayasaka, Y., \& Furukawa, T. A. (2015). Protocol registration and selective outcome reporting in recent psychiatry trials: new antidepressants and cognitive behavioural therapies. Acta psychiatrica Scandinavica, 132(6), 489-498.

https://doi.org/10.1111/acps.12502.

Smaïl-Faugeron, V., Fron-Chabouis, H., \& Durieux, P. (2015). Clinical trial registration in oral health journals. Journal of dental research, 94(3 Suppl), 8S-13S. https://doi.org/10.1177/0022034514552492.

Su, C. X., Han, M., Ren, J., Li, W. Y., Yue, S. J., Hao, Y. F., \& Liu, J. P. (2015). Empirical evidence for outcome reporting bias in randomized clinical trials of acupuncture: comparison of registered records and subsequent publications. Trials, 16, 28. https://doi.org/10.1186/s13063-014-0545-5.

Suñé, P., Suñé, J. M., \& Montoro, J. B. (2013). Positive outcomes influence the rate and time to publication, but not the impact factor of publications of clinical trial results. PloS one, 8(1), e54583. https://doi.org/10.1371/journal.pone.0054583.

Tedesco, T. K., Gimenez, T., Floriano, I., Montagner, A. F., Camargo, L. B., Calvo, A., Morimoto, S., \& Raggio, D. P. (2018). Scientific evidence for the management of dentin caries lesions in pediatric dentistry: A systematic review and network meta-analysis. PloS one, 13(11), e0206296.

https://doi.org/10.1371/journal.pone.0206296.

Wayant, C., Scheckel, C., Hicks, C., Nissen, T., Leduc, L., Som, M., \& Vassar, M. (2017). Evidence of selective reporting bias in hematology journals: A systematic review. PloS one, 12(6), e0178379.

https://doi.org/10.1371/journal.pone.0178379.

\section{Tables}

Tables 1-4 are available in the Supplemental Files section

\section{Figures}




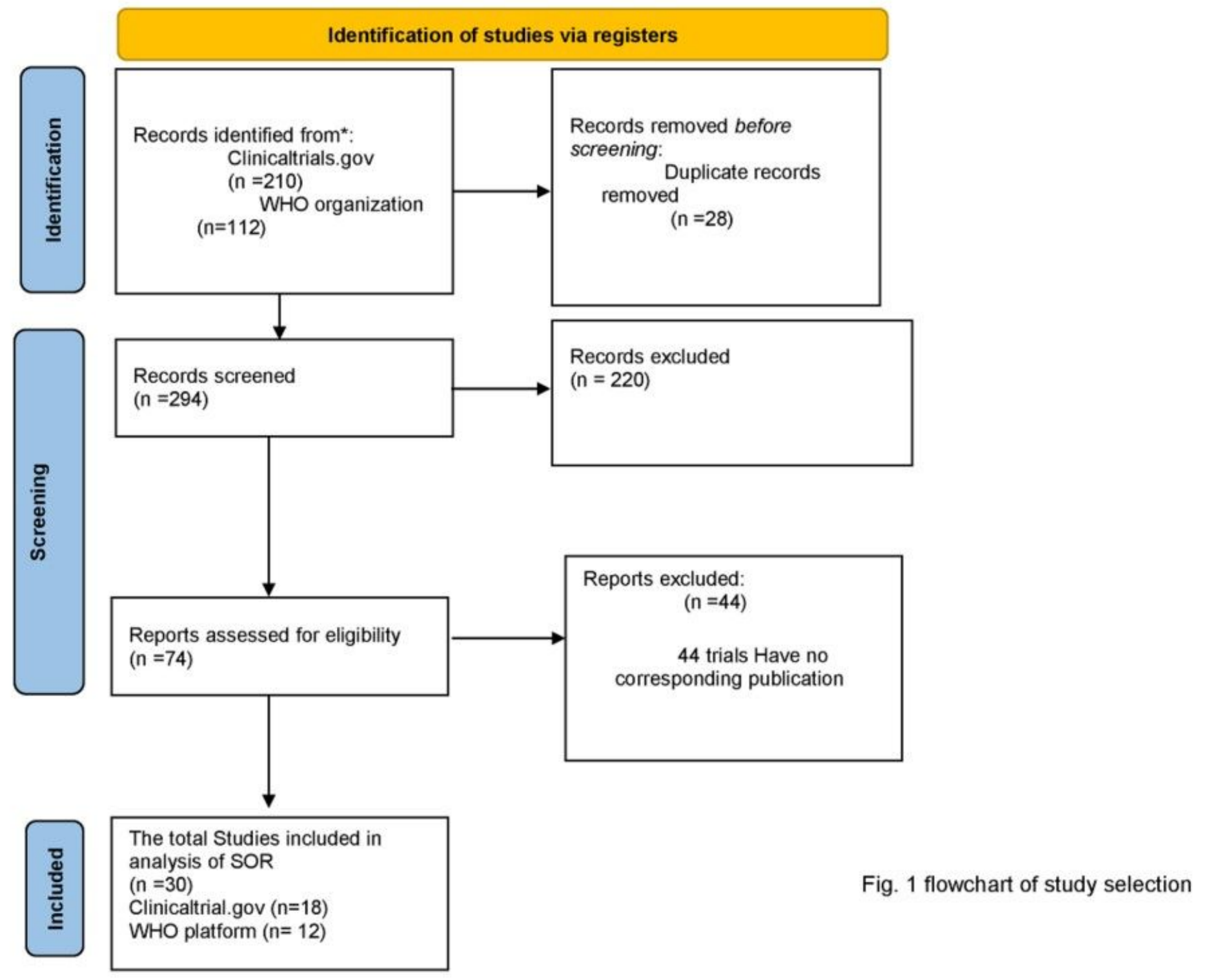

\section{Figure 1}

See image above for figure legend.

\section{Supplementary Files}

This is a list of supplementary files associated with this preprint. Click to download.

- Table1.pdf

- Table2.pdf

- Table3.pdf

- Table4.pdf 